\title{
Solving the ARE Symbolically
}

\author{
KRISTER FORSMAN and JAN ERIKSSON \\ Deptartment of Electrical Engineering \\ Linköping University \\ S-581 83 Linköping, Sweden \\ email: krister@isy.liu.se \\ 1993-03-08
}

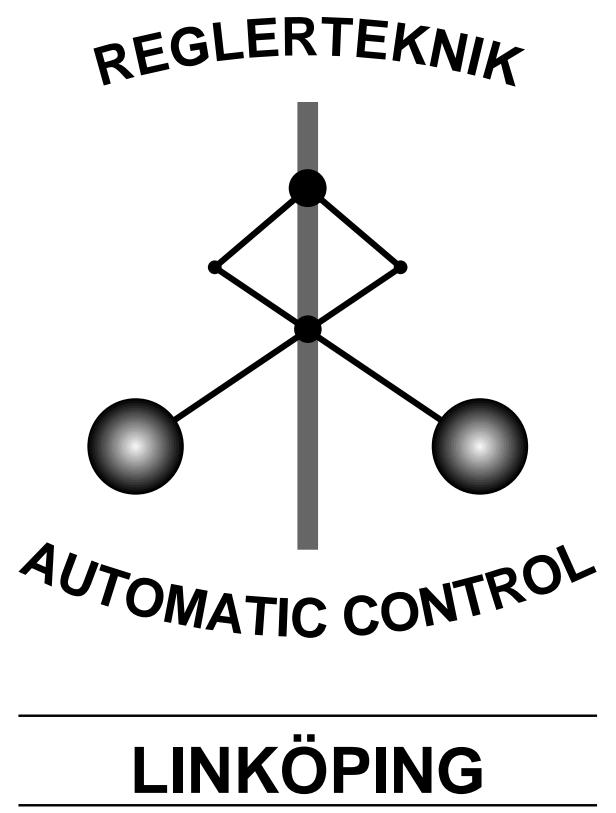

Technical reports from the automatic control group in Linköping are available by anonymous ftp at the address 130.236.24.1 (joakim.isy.liu.se). This report is contained in the compressed Postscript file named /pub/reports/LiTH-ISY-R-1456.ps.Z 


\title{
Solving the ARE Symbolically
}

\author{
Krister Forsman ${ }^{1}$ and JAN ERIKSSON \\ Department of Electrical Engineering \\ Linköping University \\ S-581 83 Linköping \\ Sweden \\ email: krister@isy.liu.se
}

1993-03-08

\begin{abstract}
Methods from computer algebra, mostly so called Gröbner bases from commutative algebra, are used to solve the algebraic Riccati equation (ARE) symbolically. The methods suggested allow us to track the influence of parameters in the system or penalty matrices on the solution. Some non-trivial aspects arise when addressing the problem from the point of view commutative algebra, for example the original equations are rational, not polynomial. We explain how this can be dealt with rather easily. Some methods for lowering the computational complexity are suggested and different methods are compared regarding efficiency. Preprocessing of the equations before applying Gröbner bases can make computations more efficient.
\end{abstract}

Keywords: algebraic Riccati equations, nonlinear matrix equations, polynomial equation systems, Gröbner bases, elimination, symbolic computation, commutative algebra, computer algebra, real algebraic geometry, nonlinear equation solving

\section{Introduction}

The algebraic Riccati equation (ARE) is probably one of the most well known and thoroughly studied nonlinear matrix equations in engineering. It arises in different aspects of optimal control and estimation problems, see e.g. [1,3, 23, 24] etc. A lot of research effort has been devoted to finding stable and fast numerical algorithms for solving the ARE, see e.g. [5, 25, $26,30]$.

In this paper we discuss how computer algebra can be used to solve the ARE symbolically. By solving we mean triangulating the system of polynomial equations that is rendered by considering each matrix entry of the matrix equation. Thus variables are eliminated successively so that we eventually obtain an equation in one variable only. One way of doing this is to compute a called Gröbner base.

An advantage with solving equations symbolically instead of numerically is that one can keep some parameters in the original equations to see how they influence the solution. A disadvantage is that symbolic solution sometimes is more expensive from a computational point of view; this is at least the case when the original problem has such a rich structure as the ARE. Some methods to decrease computational complexity will be discussed in detail.

It seems that the research presented here is original. A thorough search in the four databases INSPEC, Pascal, NTIS and Mathematics (encompassing e.g. AMS Mathematical

\footnotetext{
${ }^{1}$ All correspondence to the first author $(\mathrm{KF})$.
} 
Reviews), covering material published before the fall of 1992, for the intersection between \{ Riccati equation $\}$ and \{ Gröbner bases or standard bases or elimination or symbolic computation or computer algebra $\}$ gave zero hits.

The reader is supposed to be familiar with some basic commutative algebra, such as polynomial ring, ideal, prime ideal and dimension of an ideal. One standard reference is [4]. Hopefully the important ideas of the paper are understandable also for those that are not very familiar with this kind of mathematics. Previous knowledge of Gröbner bases is not presupposed - a brief exposition of this theory is given in section 2 .

A comment on notation: the ideal generated by the set $f_{1}, \ldots, f_{m}$ is written $\left\langle f_{1}, \ldots, f_{m}\right\rangle$. GB stands for Gröbner base (definition 2.4), and BGK for the Boege-Gebauer-Kredel algorithm (section 2.2). plex is an abbreviation for purely lexicographic and revlex for reverse graded lexicographic (definition 2.1) and g.p. stands for generic (or general) position (definition 2.5).

\section{Symbolic Equation Solving}

By solving a system of nonlinear equations we will mean triangulating it, i.e. elimination of variables as far as possible. Elimination consists in finding the elements of an ideal that belong to a certain subring. For example we may wish to find $\mathfrak{a} \cap k[S]$ for an ideal $\mathfrak{a} \subseteq k[S, T]$ where $S, T$ are sets of variables. One way of doing elimination is to compute a so called Gröbner base (GB) of an ideal w.r.t. the purely lexicographic term-ordering. Gröbner bases were defined by the Austrian mathematician Bruno Buchberger in his PhD-thesis in 1965. They are sometimes called standard bases. The computer algebra programs Maple, Reduce and Macsyma all have packages for computing GB:s.

\subsection{Basic Definitions}

Gröbner bases are special kinds of generating sets for polynomial ideals, having some appealing properties ${ }^{1}$. For a thorough introduction to GB see e.g. [9, 17, 19, 27]. This section is gives the basic definitions in the theory of Gröbner bases.

We use the multi-degree notation for the monomials of $k\left[X_{1}, \ldots, X_{n}\right]$ :

$$
\alpha \in \mathbb{N}^{n} \quad \Rightarrow \quad X^{\alpha}=X_{1}^{\alpha_{1}} \ldots X_{n}^{\alpha_{n}}, \quad \alpha=\operatorname{deg} X^{\alpha}, \quad|\alpha|=\sum_{i=1}^{n} \alpha_{i}
$$

Definition 2.1 A term ordering $<$ is a well ordering on $\mathbb{N}^{n}$ that satisfies

$$
\forall \alpha, \beta, \gamma \in \mathbb{N}^{n}: \quad \alpha<\beta \Rightarrow \alpha+\gamma<\beta+\gamma
$$

and $0<\alpha$ for all $\alpha$. Important examples are:

- the (pure) lexicographic term ordering, abbreviated plex, defined by

$$
\alpha<\beta \quad \Longleftrightarrow \quad \exists j: \alpha_{j}<\beta_{j}, \quad \forall i<j: \alpha_{i}=\beta_{i}
$$

\footnotetext{
${ }^{1}$ Using the word "base" for a generating set is somewhat obsolete. In modern terminology an ideal cannot have a basis since it is not a free module, in general.
} 
- the reverse graded lexicographic term ordering (revlex), defined by $\alpha<\beta \Longleftrightarrow$

$$
|\alpha|<|\beta| \vee\left(|\alpha|=|\beta| \wedge \exists j: \alpha_{j}>\beta_{j}, \forall i>j: \alpha_{i}=\beta_{i}\right)
$$

The notion of degree can be extended to polynomials by the convention

$$
f=\sum c_{\gamma} X^{\gamma} \Rightarrow \operatorname{deg} f=\max \left\{\gamma ; c_{\gamma} \neq 0\right\}
$$

where max refers to a given term ordering. The concept of ranking comes in handy when talking about different plex term-orderings on $k\left[X_{1}, \ldots, X_{n}\right]$ :

Definition 2.2 A ranking of the variables $X_{1}, \ldots, X_{n}$ is only a permutation of these symbols. If $a$ precedes $b$ in the permutation we say that $a$ has a lower rank than $b$, written $a<b$. If $A$ and $B$ are two sets of variables and all elements of $A$ have lower rank than all elements of $B$ we write $A<B$.

The ranking can be thought of as a reordering of the entries in the exponent vector; if $r$ is a permutation of the numbers $1, \ldots, n$ we have a corresponding ranking of the variables $X_{1}, \ldots, X_{n}$ defined by $X_{r(1)}>X_{r(2)}>\ldots>X_{r(n)} \quad \Rightarrow \quad X^{\alpha}=X_{r(1)}^{\alpha_{1}} \ldots X_{r(n)}^{\alpha_{n}}$

Definition 2.3 If we write $f=\sum c_{\gamma} X^{\gamma}$ then the leading monomial of $f$, denoted $\operatorname{lm} f$, is $X^{\operatorname{deg} f}$.

Let $\mathfrak{a}$ be an ideal in $k\left[X_{1}, \ldots, X_{n}\right]$. We define the ideal $\operatorname{lm} \mathfrak{a}=\langle\{\operatorname{lm} f ; f \in \mathfrak{a}\}\rangle$.

Definition 2.4 The set $\mathbf{G} \subseteq \mathfrak{a}$ is a Gröbner base for $\mathfrak{a}$ (w.r.t. a given term ordering) iff $\operatorname{lm} \mathfrak{a}=\langle\operatorname{lm} \mathbf{G}\rangle$.

It can be showed that every ideal possesses a finite Gröbner basis, which is unique, under some weak assumptions. In the mid-70's Gröbner bases were discovered to have the following elimination property, which is the reason for our interest in them:

Theorem 2.1 Let $\mathfrak{a}$ be an ideal in $k\left[X_{1}, \ldots, X_{n}\right]$ and partition $X_{1}, \ldots, X_{n}$ into two disjoint sets $S$ and $T$. If $\mathbf{G}$ is a plex $G B$ for $\mathfrak{a}$ w.r.t. some ranking $S<T$ then $\mathbf{G} \cap k[S]$ is a $G B$ for $\mathfrak{a} \cap k[S]$.

Proof. See e.g. [19] or [9].

In words, theorem 2.1 states that with a proper choice of ranking the Gröbner base $\mathbf{G}$ of $\mathfrak{a}$ tells us if there are elements in $\mathfrak{a}$ that are polynomials in the variables $S$ only, and even more: $\mathbf{G}$ contains a generating set of the subideal in question.

There is an algorithm, due to Buchberger, that computes the GB of any ideal, given a finite set of generators [8]. The algorithm has been implemented on computers in several versions; the symbolic algebra programs Maple [10], Axiom, Macsyma and Reduce all have GB packages. The following theorem explains how GB can be used equation solving:

Theorem 2.2 Let $\mathfrak{a}$ be an ideal in $k\left[X_{1}, \ldots, X_{n}\right]$. We have that $\operatorname{dim} \mathfrak{a}=0$ iff for all $i$ there is a $p \in G B(\mathfrak{a})$ such that $\operatorname{lm} p \in k\left[X_{i}\right]$. 
Proof. See [19] or [9, page 209].

If the polynomials in $\mathfrak{a}$ have finitely many zeroes in common, then a GB for $\mathfrak{a}$ w.r.t. the ranking $X_{1}<X_{2}<\ldots<X_{n}$ contains a polynomial $p_{1}$ in $X_{1}$ the roots of which we can use numerical methods to determine. Furthermore there is a polynomial $p_{2}$ such that $\operatorname{lm} p_{2} \in k\left[X_{2}\right]$. We must have $p_{2} \in k\left[X_{1}, X_{2}\right]$ according to theorem 2.1. Now we substitute the different values of $X_{1}$ satisfying $p_{1}=0$ into $p_{2}$ to get the values of $X_{2}$, etc.

Theorem 2.2 does not state that there are $n$ elements in the GB: the set $\left\{X_{1}^{2}, X_{1} X_{2}, X_{2}^{2}\right\}$ is a $\mathrm{GB}$ of a zero-dimensional ideal in $k\left[X_{1}, X_{2}\right]$. For prime ideals the situation is simpler, though: a plex GB for a prime zero-dimensional ideal in $k\left[X_{1}, \ldots, X_{n}\right]$ has $n$ elements [19].

In [19] it is proved that the generic look of a plex GB for a zero-dimensional ideal w.r.t. $X_{n}<\ldots<X_{1}$ is

$$
\left\{X_{1}-p_{1}, X_{2}-p_{2}, \ldots, X_{n-1}-p_{n-1}, p_{n}\right\}
$$

where $p_{i} \in k\left[X_{n}\right]$ for all $i$, and $\operatorname{deg} p_{n}>\operatorname{deg} p_{i}$ for $i=1, \ldots, n-1$.

Definition 2.5 An (arbitrary) ideal that has a GB of the type (2) w.r.t. the plex termordering is said to be in generic position.

Thus a zero-dimensional ideal is in generic position (g.p.) if for two different zeroes $\left(a_{1}, \ldots, a_{n}\right)$ and $\left(b_{1}, \ldots, b_{n}\right)$ we have $a_{n} \neq b_{n}$. In [19] it is proved that almost all linear transformations of a simple type put a zero-dimensional ideal in g.p. Yet another formulation is: a prime zero-dimensional ideal is in g.p. iff the GB provides a primitive element of the algebraic field extension defined by the ideal.

\subsection{Computational Complexity and the BGK Algorithm}

One disadvantage with plex Gröbner bases is that the complexity for computing them is in general very high. Now, it has been showed that the complexity for computing a GB for total degree orderings, e.g. revlex, is in general lower than in the case of a plex ordering.

There is an approach to elimination theory that is also based on Gröbner bases, but does not use the plex term ordering. This approach is primarily due to Boege, Gebauer and Kredel [7]; it is also discussed in e.g. [9, 18]. The algorithm, let us call it the BGK algorithm, gives the univariate polynomial that generates the contraction $I \cap k\left[X_{i}\right]$ for some $X_{i}$. Thus, in particular, it works for all zero-dimensional ideals. We call the polynomial obtained the minimal polynomial of $X_{i}$.

The idea is first to compute a Gröbner base $\mathbf{G}$ w.r.t. a revlex ordering and then compute remainders $R_{d}=\operatorname{rem}\left(X_{i}^{d}, \mathbf{G}\right)$ for successive $d:$ s. Each $R_{d}$ is considered as an element in the $k$-vector space of forms of degree $d$ in $k\left[X_{1}, \ldots, X_{n}\right]$. For $d$ large enough the expressions for $R_{0}, \ldots, R_{d}$ are linearly dependent over $k$. This linear dependency relation gives us the minimal polynomial for $X_{i}$.

The reason for using the approach described above is thus that it is typically more efficient than computing a plex GB; see [7]. The BGK algorithm is part of the Gröbner base package in Maple, available under the name finduni.

If the ideal considered is in generic position then the minimal polynomial obtained from BGK tells us how many zeroes there are and whether they are real or not.

It is possible to determine if an ideal is g.p. or not without computing a plex GB. If $I$ is zero-dimensional then the dimension of the $k$-vector space $k\left[X_{1}, \ldots, X_{n}\right] / I$, i.e. the Hilbert 
series evaluated at 1 , can be computed from any GB of $I$. If this number is equal to the degree of the minimal polynomial for $X_{n}$ then $I$ is in g.p.

\subsection{Comparison with Other Symbolic Methods}

As mentioned there are some advantages of using symbolic instead of numerical methods for equation solving. But there are other methods than GB for solving systems of polynomial equations symbolically. Let us briefly discuss the most obvious alternatives:

- Resultants are probably the oldest tools in elimination theory. They are based on the idea of converting the nonlinear system to a linear one by multiplying the original equations by suitable monomials and considering the polynomials as elements in a linear space spanned by some monomials. Originally they were defined to deal with arbitrary many equations in arbitrary many variables, but today the word resultant often refers to the determinant of the Sylvester matrix which is a reformulation for the case of two equations. Resultants sometimes more convenient than GB for solving two equations in two variables. If the number of equations is larger than two, they often turn out (empirically) to be less efficient than GB. Another disadvantage with resultants are the so called parasitic solutions that occur. References: [17, 20, 22].

- Characteristic sets provide another method for triangulating systems of polynomial equations. It has been proved that for prime ideals characteristic sets can be viewed as a special case of GB via localization methods; see e.g. [11, 15]. It is sometimes claimed that characteristic sets are more efficient than GB from a computational point of view, but it is questionable if this is still true when the "tricks" described in [15] (basically, BGK and localization to rings with rational function coefficient fields) are used before applying Buchberger's algorithm. Furthermore, it seems hard, if not impossible, to implement algorithms that compute characteristic sets for arbitrary (not necessarily prime) ideals. References: e.g. [11, 16, 21, 29].

\subsection{Inequations}

It is possible to include inequations in the framework of commutative algebra: if we wish to consider the system

$$
f_{1}=0, \ldots, f_{m}=0, q \neq 0
$$

where $f_{i}, q \in k\left[X_{1}, \ldots, X_{n}\right]$ we may study the ideal

$$
\left\langle f_{1}, \ldots, f_{m}, Z q-1\right\rangle \subseteq k\left[X_{1}, \ldots, X_{n}, Z\right]
$$

where $Z$ is an auxiliary variable; see e.g. [13]. This is known as the Rabinovich trick. When studying a system of equations with rational left hand sides

$$
\frac{p_{i}}{q_{i}}=0, \quad p_{i}, q_{i} \in k\left[X_{1}, \ldots, X_{n}\right] \quad i=1, \ldots, m
$$

we could of course consider the ideal $\left\langle p_{1}, \ldots, p_{m}\right\rangle$. But if we wish to exclude solutions $x$ such that $q_{j}(x)=0$ for some $j$ we can use the Rabinovich trick, and instead study the ideal $\left\langle p_{1}, \ldots, p_{m}, Z q_{1} \ldots q_{m}-1\right\rangle$ in $k\left[X_{1}, \ldots, X_{n}, Z\right]$. 


\section{The Algebraic Riccati Equation}

Let us now see how the ARE can be solved using symbolic manipulation and methods from commutative algebra.

\subsection{Generalities}

Consider a continuous or discrete time, stochastic, time-invariant control system written in state space form:

$$
\dot{x}(t)=A x(t)+b u(t)+v(t), \quad y(t)=c x(t)+e(t)
$$

or

$$
x(t+1)=A x(t)+b u(t)+v(t), \quad y(t)=c x(t)+e(t)
$$

where $v, e$ are conventional white noises with covariance matrices $R_{1}, R_{2}$, respectively. For the continuous time LQ optimization problem the ARE is

$$
A^{T} S+S A+Q_{1}-S b Q_{2}^{-1} b^{T} S=0
$$

where superscript $T$ denotes transpose, and in discrete time it is

$$
A^{T} S A+Q_{1}-A^{T} S b\left(b^{T} S b+Q_{2}\right)^{-1} b^{T} S A=S
$$

For the continuous time optimal filtering problem the ARE is

$$
A P+P A^{T}+R_{1}-P c^{T} R_{2}^{-1} c P=0
$$

and in discrete time

$$
A P A^{T}+R_{1}-A P c^{T}\left(c P c^{T}+R_{2}\right)^{-1} c P A^{T}=P
$$

The unknown matrices $P, S$ are symmetric in all cases. Observability-type conditions ensure the existence of positive semidefinite $P, S$ satisfying one of (8) - (11); see the references.

We now wish to consider the ARE as a system of polynomial equations $e_{i, j}=0$, one for each matrix entry, modulo entries that are identical due to the symmetry of $P$. For simplicity we assume that the system is SISO, so that we only have one denominator. Letting the polynomial $f$ represent the condition that the denominator $\neq 0$ via the Rabinovich trick we get the ideal

$$
\mathfrak{a}=\left\langle e_{i, j}, f\right\rangle_{\substack{i=1, \ldots, n \\ j \geq i}}
$$

generated by $\frac{1}{2} n(n+1)+1$ polynomials. The ideal $\mathfrak{a}$ is the main object of study in this paper. If $p_{i, j}$ are the elements of $P$ and $z$ is the Rabinovich variable then $\mathfrak{a}$ lives in the ring

$$
k\left[z, p_{i, j}\right]_{\substack{i=1, \ldots, n \\ j \geq i}}
$$

where $k$ is some field. The most interesting case for symbolic computation is of course when $k$ contains rational functions of some parameters occurring either in the covariance or penalty matrices or in the system model.

In the sections below we outline three different GB approaches for solving the ARE symbolically. In section 4 we will see how the algorithms work on some concrete examples. 


\subsection{Standard Gröbner Base Solution}

The most straightforward idea is to apply lexicographic GB directly to the ideal $\mathfrak{a}$ defined in equation (12). According to theorem 2.2 this gives us information about the possible solutions of the ARE, since there is a finite number of solutions to the ARE under the conditions above.

This means that e.g. if we choose a lexicographic term ordering ranking $p_{1,1}$ lowest, then $G B(\mathfrak{a})$ will contain the minimal polynomial for $p_{1,1}$. If there are parameters present the minimal polynomial will reveal how these influence $p_{1,1}$ and thereby the Kalman, or LQ, gain.

An extra difficulty in the commutative algebraic framework is that it is not immediately obvious which value of $p_{1,1}$ that renders a positive definite solution, unless the minimal polynomial of $p_{1,1}$ has only one positive real root, of course. The positivity issue has to be handled separately, using e.g. Sylvester's subdeterminant criterion.

\subsection{BGK Solution}

Practical experience indicates that $\mathfrak{a}$ is in generic position, which makes the BGK algorithm quite suitable (see section 2.2). If we determine the minimal polynomial for some diagonal element $p_{i, i}$ and find that it has only one positive root then we know what value $p_{i, i}$ has to take. If not we will have to compute minimal polynomials for the other variables as well, to see which combination of solutions that gives a positive definite $P$-matrix. A complete algorithm for solving the ARE using BGK thus looks as follows:

1. Find the minimal polynomial $m_{i, j}$ for each one of the variables $p_{i, j}$ using BGK.

2. Determine the roots of each $m_{i, j}$.

3. Check combinations of roots of the $m_{i, j}$ to see which one renders a positive definite $P$-matrix.

Note that this algorithm only requires one GB calculation - the $m_{i, j}$ are computed using remaindering on this GB and linear algebra. An intelligent search in step 3 hopefully saves us from testing all combinations of roots; for example we know that all $p_{i, i}$ have to be positive.

\subsection{Putting the System in Canonical Form}

In this section we will indicate how some preprocessing the equations can improve the symbolic solving of the equations.

The idea is first to put the linear system (6) or (7) in some canonical form. This makes the ARE have a particularly appealing shape: many of the equations become linear in some of the variables. If we use the linearity to eliminate these variables ourselves before handing over to Buchberger's algorithm it seems that we can save computation time. This approach is inspired by [28], but the cited work cannot be applied directly in the symbolic case [12].

Some investigations have been made regarding in what order these (partly) linear equations should be used when eliminating the variables. Unfortunately, this combinatorial problem is too complex to be discussed here; details can be found in [12]. Let $n$ be the number

of variables, so that there are $\frac{1}{2} n(n+1)+1$ original equations. For $n \leq 6$ the elimination procedure described above reduces the number of equations to $n$ for even $n$, and $\frac{1}{2}(n+1)$ for odd $n$. The remaining equations are nonlinear in all their variables. This result has not been proved for an arbitrary $n$. 
As a first, empirical result it seems that this method is the most efficient one of those mentioned here. This is true even if we include the cost of transforming a linear system to canonical form in the total time consumption.

\section{Examples}

Let us now see how the methods suggested work on some concrete examples. The computations described below were all performed in Maple. The GB package in Maple is not the most efficient one available, but there are other advantages with Maple, e.g. that the BGK algorithm is available.

We start by a simple example, where the solution can actually be computed by hand.

Example 4.1 Consider the discrete time second order system

$$
x(t+1)=\left[\begin{array}{ll}
1 & 1 \\
0 & 0
\end{array}\right] x(t)+\left[\begin{array}{l}
0 \\
1
\end{array}\right] u(t)
$$

We are interested in minimizing the criterion

$$
J=\sum_{0}^{\infty}\left\{q_{1} x_{1}^{2}(t)+q_{2} x_{2}^{2}(t)+u(t)^{2}\right\}
$$

This LQ problem gives the ARE (9), which corresponds to an ideal $\mathfrak{a}$ in the ring

$$
\mathbb{Q}\left(q_{1}, q_{2}\right)\left[z, s_{1,1}, s_{1,2}, s_{2,2}\right]
$$

defined by

$$
\begin{aligned}
& \mathfrak{a}=\left\langle q_{1} s_{2,2}+q_{1}-s_{1,2}^{2}, \quad s_{1,1} s_{2,2}+s_{1,1}-s_{1,2} s_{2,2}-s_{1,2}-s_{1,2}^{2},\right. \\
& \left.s_{1,1} s_{2,2}+s_{1,1}-s_{2,2}^{2}-s_{2,2}+q_{2} s_{2,2}+q_{2}-s_{1,2}^{2}, \quad z s_{2,2}+z-1\right\rangle
\end{aligned}
$$

The Maple command, once the grobner package has been loaded, for computing the plex GB for $\mathfrak{a}$ w.r.t. the ranking $z>s_{2,2}>s_{1,2}>s_{1,1}$ is

$\operatorname{gbasis}(F,[z, s[2,2], s[1,2], s[1,1]], p l e x)$ :

where $\mathrm{F}$ is the list of generators of $\mathfrak{a}$ given in (16). The GB for $\mathfrak{a}$ is

$$
\begin{gathered}
\left\{\left(1+2 q_{2}+q_{2}^{2}\right) z-2 q_{1}-1-q_{2}+s_{1,1}, s_{2,2}-s_{1,1}+q_{1}-q_{2},\right. \\
\left.s_{1,2}-s_{1,1}+q_{1}, s_{1,1}^{2}-3 q_{1} s_{1,1}-q_{1}-q_{1} q_{2}+2 q_{1}^{2}\right\}
\end{gathered}
$$

Thus $\mathfrak{a}$ is in generic position and the minimal polynomial for $s_{1,1}$ is

$$
m_{1,1}=s_{1,1}^{2}-3 q_{1} s_{1,1}-q_{1}-q_{1} q_{2}+2 q_{1}^{2}
$$

Since $\mathfrak{a}$ is in g.p. it suffices $m_{1,1}$ has only one positive root for us to know that this root gives wanted $S$-matrix. So e.g. if $q_{1}=1, q_{2}=4$ we get that $s_{1,1}=3.79$, and back-substituting we find that $s_{1,2}=2.79$ and $s_{2,2}=6.79$.

The following example is already impossible to work through by hand: 
Example 4.2 Consider the LQ problem for a continuous time system given by

$$
A=\left[\begin{array}{ccc}
a_{1}+a_{2}-1 & a_{1}+a_{2}-a_{1} a_{2} & -a_{1} a_{2} \\
1 & 0 & 0 \\
0 & 1 & 0
\end{array}\right], \quad b=\left[\begin{array}{l}
1 \\
0 \\
0
\end{array}\right]
$$

i.e. a third order system with poles in $-1, a_{1}$ and $a_{2}$, with penalty matrices $Q_{1}=3 \times 3$-identity matrix and $Q_{2}=1$. Proceeding as in example 4.1 we find that also in this case $\mathfrak{a}$ is in g.p. and that the minimal polynomial $m_{1,1}$ for $s_{1,1}$ is dense and of degree 8 . Its first few terms are

$$
s_{1,1}^{8}+8\left(1-a_{1}-a_{2}\right) s_{1,1}^{7}+\left(20+24 a_{1}^{2}+56 a_{1} a_{2}-56 a_{1}+24 a_{2}^{2}-56 a_{2}\right) s_{1,1}^{6}+\ldots
$$

The size of the Maple object representing $m_{1,1}$ is 1.4 kbytes. It took approximately 5 seconds to compute the GB for $\mathfrak{a}$ on a SUN Sparc 2 with 32 Mbytes of memory.

Example 4.3 Consider discrete time system given by

$$
A=\left[\begin{array}{ccc}
a_{1}+a_{2}+\frac{1}{2} & -\frac{1}{2} a_{1}-\frac{1}{2} a_{2}-a_{1} a_{2} & \frac{1}{2} a_{1} a_{2} \\
1 & 0 & 0 \\
0 & 1 & 0
\end{array}\right], \quad b=\left[\begin{array}{l}
1 \\
0 \\
0
\end{array}\right]
$$

i.e. a third order system with poles $\frac{1}{2}, a_{1}, a_{2}$. We wish to solve the LQ-problem with penalty matrices as in example 4.2. This problem is computationally much harder than example 4.2, despite the superficial resemblance. Using the same computer as in the previous example, it takes about 1.5 hours to compute a plex GB for the ideal $\mathfrak{a}$. This GB occupies 120 kbytes and it shows that $\mathfrak{a}$ is in g.p. Determining a plex GB for $\mathfrak{a}$ and then finding minimal polynomials for all $s_{i, j}$ using BGK is somewhat faster in this example: about $45 \mathrm{~min}$. Of this time, only one minute is spent computing the GB. It remains to be checked if the other time is mostly spent computing remainders or solving linear equation systems.

\section{$5 \quad$ Future Research}

Above we have presented some first investigations on how Gröbner bases and commutative algebra can be applied when solving the ARE. There are many possible future directions of this research. Some open questions have been touched upon in section 3, e.g:

- Is a always in generic position?

- Is it always possible to reduce the number of nonlinear equations by putting the linear system in canonical form?

The problem of singling out the only positive definite solution of the ARE belongs to real algebraic geometry, q.v. $[2,6]$. It is a natural next step to check if there are systematic and efficient methods for handling the positivity problem within the existing theory of this field. There are many obvious ways that can be taken, but one has to be very careful in order not to let the computational complexity grow unacceptably large.

In the present work the ARE is interpreted as a system of polynomial equations - no use is made of the original matrix structure. It might be that the algorithm for computing GB 
can be modified in order to take the special structure into account. No such method is known to the authors at the moment, and if there is such a method it is not immediately evident.

Another interesting approach is the possibility of combining numerical methods for equation solving with symbolical ones. For systems without the particular matrix background such combinations are sometimes advantageous [14].

\section{Acknowledgement}

This work was financially supported by the Swedish Council for Technical Research (TFR).

\section{References}

[1] B.D.O. Anderson and J.B. Moore. Optimal Filtering. Prentice Hall, 1979.

[2] D.S. Arnon and B. Buchberger, editors. Algorithms in Real Algebraic Geometry. Academic Press, 1988. Reprint from J. Symbolic Computation, vol. 5:1-2.

[3] K.J. Åström and B. Wittenmark. Computer Controlled Systems: Theory and Design. Prentice-Hall, international edition, 1984.

[4] M.F. Atiyah and I.G. MacDonald. Introduction to Commutative Algebra. AddisonWesley, 1969.

[5] G. Bierman. Factorization Methods for Discrete Estimation. Academic Press, 1977.

[6] J. Bochnak, M. Coste, and M-F. Roy. Géometrie algébrique réelle. Springer, 1987.

[7] W. Boege, R. Gebauer, and H. Kredel. Some examples for solving systems of algebraic equations by calculating Gröbner bases. J. Symbolic Computation, 1:83-98, 1986.

[8] B. Buchberger. Ein algorithmisches Kriterium für die Lösbarkeit eines algebraischen Gleichungssystems. Aequationes Mathematicae, 4:374-383, 1970.

[9] B. Buchberger. Gröbner bases: An algorithmic method in polynomial ideal theory. In N.K. Bose, editor, Multidimensional Systems Theory, pages 184-232. Dordrecht Reidel, 1985.

[10] B. Char, K.O. Geddes, G.H. Gonnet, B.L. Leong, M.B. Monagan, and S.M. Watt. Maple V Library Reference Manual. Springer, 1991.

[11] S.-C. Chou. Mechanical Geometry Theorem Proving. Mathematics and Its Applications. D. Reidel, 1988.

[12] J. Eriksson and K. Forsman. Maple code for solving the ARE symbolically. Technical report, Dept. of Electrical Engineering, Linköping University, S-581 83 Linköping, Sweden, 1993. To appear.

[13] A. Ferro and G. Gallo. Gröbner bases, Ritt's algorithm and decision procedures for algebraic theories. In L. Huguet and A. Poli, editors, Applied Algebra, Algebraic Algorithms and Error-Correcting Codes, volume 356 of LNCS, pages 230-237. Springer, 1989. Proc. AAECC-5, Menorca. 
[14] K. Forsman. Constructive Commutative Algebra in Nonlinear Control Theory. PhD thesis, Dept. of Electrical Engineering, Linköping University, S-581 83 Linköping, Sweden, 1991.

[15] K. Forsman. Localization and base change techniques in computational algebra. Technical Report LiTH-ISY-R-1445, Dept. of Electrical Engineering, Linköping University, S-581 83 Linköping, Sweden, February 1993. Available by anonymous ftp 130.236.24.1.

[16] G. Gallo and B. Mishra. Efficient algorithms and bounds for Wu-Ritt characteristic sets. In T. Mora and C. Traverso, editors, Effective Methods in Algebraic Geometry, volume 94 of Progress in Mathematics, pages 119-142. Birkhäuser, 1991. From the Symposium MEGA 90, held at Castiglioncello, Italy, 1990.

[17] K.O. Geddes, S.R. Czapor, and G. Labahn. Algorithms for Computer Algebra. Kluwer Academic Publishers, 1992.

[18] P. Gianni and T. Mora. Algebraic solution of systems of polynomial equations using Gröbner bases. In L. Huguet and A. Poli, editors, Applied Algebra, Algebraic Algorithms and Error-Correcting Codes, volume 356 of LNCS, pages 247-257. Springer, 1989. Proc. AAECC-5, Menorca.

[19] P. Gianni, B. Trager, and G. Zacharias. Gröbner bases and primary decomposition of polynomial ideals. In L. Robbiano, editor, Computational Aspects of Commutative Algebra, pages 15-33. Academic Press, 1989. From J. Symb. Comp. Vol. 6, nr. 2-3.

[20] W. Gröbner. Moderne algebraische Geometrie. Die Idealtheoretischen Grundlagen. Springer, 1949.

[21] L.C.G.J.M. Habets. Characteristic sets in commutative algebra: an overview. Technical Report COSOR 92-24, Department of Mathematics and Computing Science, Eindhoven University of Technology, P.O. 513, 5600 MB Eindhoven, The Netherlands, 1992.

[22] W.V.D. Hodge and D. Pedoe. Methods of Algebraic Geometry. Cambridge University Press, 1953.

[23] T. Kailath. Linear Systems. Systems sciences. Prentice-Hall, 1980.

[24] H. Kwakernaak and R. Sivan. Linear Optimal Control Systems. Wiley, 1972.

[25] A.J. Laub. A Schur method for solving algebraic Riccati equations. IEEE Trans. Aut. Contr., AC-24(6):913-921, December 1979.

[26] A.G.J. MacFarlane. An eigenvector solution of the optimal linear regulator. J. Electron. Control, 14:643-654, 1963.

[27] F. Pauer and M. Pfeifhofer. The theory of Gröbner bases. L'Enseignement Mathématique, 34:215-232, 1988.

[28] C.E.M. Pearce. On the solution of a class of algebraic matrix Riccati equations. IEEE Trans. Aut. Contr., AC-31(3), March 1986.

[29] J.F. Ritt. Differential Algebra. Dover, 1950.

[30] P. van Dooren. A generalized eigenvalue approach for solving Riccati equations. SIAM J. Sci. Stat. Comp., 2:121-135, 1981. 